Geophysical Research Abstracts, Vol. 10, EGU2008-A-08565, 2008

SRef-ID: $1607-7962 /$ gra/EGU2008-A-08565

EGU General Assembly 2008

(C) Author(s) 2008

\title{
Integrating the Climate Science Modelling Language with geospatial software and services
}

\author{
D. Lowe (1), B.N. Lawrence(1), S. Pascoe (1), A. Woolf (1) \\ (1) Science and Technology Facilities Council (d.lowe@ rl.ac.uk)
}

Much consideration is rightly given to the design of metadata models to describe data. At the other end of the data-delivery spectrum much thought has also been given to the design of geospatial delivery interfaces such as OGC WCS, WMS and WFS. Our recent experience with the Climate Science Modelling Language shows that an implementation gap exists where many challenges remain unsolved. To bridge this gap requires transposing information and data from one world view of geospatial climate data to another. Some of the issues include: the loss of information in mapping to a common information model, the need to create 'views' onto file-based storage, and the need to map onto an appropriate delivery interface (as with the choice between WFS and WCS for feature types with coverage-valued properties). Here we summarise the approaches we have taken in facing up to these problems. 